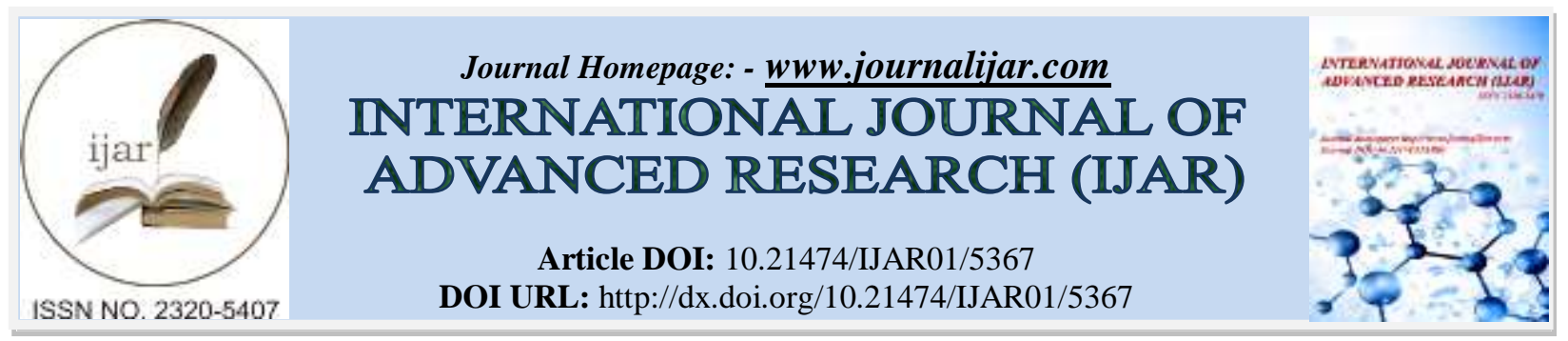

RESEARCH ARTICLE

\title{
MARINE BACTERIA AND FUNGI AS SOURCES FOR BIOACTIVE COMPOUNDS: PRESENT STATUS AND FUTURE TRENDS.
}

Varuni K Gunathilake.

Department of Zoology, Faculty of Applied Sciences, University of Sri Jayewardenepura, Gangodawila, Nugegoda, Sri Lanka.

\section{Manuscript Info}

Manuscript History

Received: 09 July 2017

Final Accepted: 11 August 2017

Published: September 2017

Key words:-

marine bacteria, marine fungi, bioactive compounds, marine pharmacognosy.

\begin{abstract}
Marine organisms undergo a vast range of chemical and physical conditions in the marine environment, thus a high diversity is reported in the bioactive compounds they produce. With the development of marine biotechnology, a considerable number of researches are focused on marine bacteria and fungi-derived bioactive compounds. As a result, marine bacteria and fungi are ranked on the top of the hierarchy of all organisms, as they are responsible for producing a wide range of bioactive secondary metabolites with potential pharmaceutical applications. Thus, they have the potential to provide future drugs against important diseases, such as cancer, a range of viral diseases, malaria, and inflammations. This review aims at describing some of the most highly cited reviews of the last decade on marine bacteria and fungi -derived bioactive compounds and the most promising substances extracted and isolated from these for pharmaceutical applications.
\end{abstract}

Copy Right, IJAR, 2017,. All rights reserved.

\section{Introduction:-}

Bacteria and fungi have a major impact on the development of medical science since the discovery that they not only are the cause of infection but also produce organic compounds that can both cure infections and help treat a variety of non infectious diseases. With the rapid development of marine biotechnology in the past few decades, marine bacteria and fungi are considered as promising agents for the discovery of bioactive natural chemical substances with respect to the diversity of their primary and secondary metabolites. Due to the fact that these organisms live in a biologically competitive environment with unique conditions of $\mathrm{pH}$, temperature, pressure, oxygen, light, nutrients and salinity, there is a high potential for those to produce metabolites exhibit special biological activities compared with terrestrial counterparts. As bacteria and fungi have rapid growth, reproduction and ability to live in ubiquitous habitats, they are considered as the most prominent members of marine and terrestrial ecosystems.

Surviving in the marine environment is challengeable as bacteria and fungi have to undergo tremendous physical and chemical pressure, thus exhibit various types of adaptations in their anatomy and physiology (Jha and Zi-rong, 2004). From an ecological point of view bacterial and fungal secondary metabolites may probably act specifically in interspecies interactions to protect the host and/or the producer against competitors and/or diseases. Further, these metabolites may play an important role in the marine habitats as they provide information on ecology of marine communities.

Corresponding Author:- Varuni K Gunathilake.

Address:- Department of Zoology, Faculty of Applied Sciences, University of Sri Jayewardenepura, 
Although only a small number of microorganisms have been investigated for bioactive metabolites, yet a huge number of active substances with some of them featuring unique structural skeletons have been isolated (Debbab,2010). Therefore, the discovery of such compounds is continuing and yet to be explored (Zhang et al, 2005).

\section{Marine Bacteria-derived bioactive compounds:-}

Marine actinomycetes, a group of gram positive bacteria, are responsible for the production of about half of the discovered bioactive secondary metabolites (Berdy J, 2005) such as antibiotics (Strohl, 2004), antitumor (Cragg et al, 2005) and immunosuppressive agents (Oldfield et al, 1998). The discovery of numerous new marine actinomycete taxa, their demonstrated metabolic activity in natural environments and ability to form stable populations in different habitats clearly illustrate that indigenous marine actinomycetes indeed exist in the oceans (Lam, 2006). Further, novel compounds with biological activities have been isolated from these marine actinomycetes, indicating that marine actinomycetes are an important source for the discovery of novel secondary metabolites (Jensen et al, 2005). Although the exploitation of marine actinomycetes as a source for discovery of novel secondary metabolites is comparatively at an early stage, numerous novel metabolites have been isolated in the past few years (Lam, 2006). Abyssomicin C which is potent inhibitor of folic acid biosynthesis, is a novel polycyclic polyketide antibiotic produced by a marine Verrucosispora strain (Riedlinger et al, 2004). Abyssomicin C possesses potent activity against Gram-positive bacteria, including clinical isolates of multiple-resistant and vancomycin-resistant Staphylococcus aureus. Abyssomicin C (Riedlinger et al, 2005) has the potential to be developed as antibacterial agent against drug-resistant pathogens.

Diazepinomicin, which is isolated from Micromonosproa sp. is a unique farnesylated dibenzodiazepinone with potential antibacterial, anti-inflammatory and anti-tumor activity (Charan et al.2004). It has a broad spectrum of in vitro cytotoxicity and also in vivo activity against glioma, breast and prostate cancer in mouse models. Salinosporamide A, a novel blactone- g-lactam isolated from a fermentation broth of a new obligate marine actinomycete, Salinispora tropica (Maldonado et al, 2005) is an orally active proteasome inhibitor that induces apoptosis in multiple myeloma cells with mechanisms distinct from the commercial proteasome inhibitor anticancer drug Bortezomib (Chauhan et al, 2005). Salinospramide A is also the first clinical candidate for the treatment of cancer produced by saline fermentation of an obligate marine actinomycete (Lam, 2006).Anti bacterial compounds such as Abyssomicins have been isolated from Verrucosispora sp while Bonactin, Friyocyclinone, Glaciapyrroles, Glutingimycinand Helquinoline have been isolated from Streptomyces sp (Lam et al, 2005). Bonactin exhibits both anti bacterial and anti fungal properties. Trioxacarcins, isolated from Streptomyces sp contain anti bacterial, anti cancer and anti malarial properties (Lam et al, 2005).

Unlike their terrestrial counterparts, marine bacteria undergo harsh oceanic climates and seasonal and temporal changes in physiochemical parameters. They also produce some secondary metabolites for their defence against other microorganisms and these secondary metabolites serve as a source of bioactive compounds for use in human therapies (Bhatnagar and Kim, 2010).

Compounds isolated from Pseudomonas which are gram-negative Gamma proteo bacteria have showed potent antimicrobial activities (Bhatnagar and Kim, 2010). Further, bioactive compounds such as pyrroles, pseudopeptide pyrrolidinedione, phloroglucinol, phenazine, benzaldehyde, quinoline, quinolone, phenanthren, phthalate, andrimid, moiramides, zafrin and bushrin have been isolated from a wide range of gram negative bacteria (Romanenko et al, 2008). Various anti fungal activity of extracts were reported in Stenotrophomonas which was the first report on anti microbial activity from bacterial strains associated with deep sea invertebrates (Bhatnagar and Kim, 2010). Heterotrophic bacterial strains isolated from marine ark shell Anadara broughtoni have revealed antimicrobial and hemolytic activities (Romanenko et al, 2008). Cultures of the marine bacterial isolate Brevibacillus laterosporus (strain PNG276) yielded new lipopeptide named tauramamide and ethyl esters, which showed potent inhibitory activity against the important Gram positive human pathogen Enterococcus sp (Debbab et al,2010) . Pseudomonas stutzeri (strain CMG 1030) that showed pronounced inhibitory activity against several pathogenicbacteria, including MRSA strains (Debbab et al, 2010). Chemical investigation of the ethyl acetate extract yielded a new antibacterial metabolite named zafrin which indicated activity against a panel of clinically important microorganisms such as Bacillus subtilis was faster than for ampicillin, vancomycin or tetracycline (Debbab et al, 2010). Chemical; compounds isolated from the new bacterial genus Marinispora (strain NPS008920) have shown a broad spectrum of antimicrobial activities, similar to those of the commercial antibiotic linezolid (Zyvox) (Barbachyn and Ford, 
2003). Further, compounds isolated from these bacteria shows a broad spectrum of anti bacterial activity (Debbab et al, 2010).

Another marine bacterial species Pseudoalteromonasis is also responsible for producing a considerable number of bioactive compounds. Egan and coworkers (2002) have reported that the yellow pigment of Pseudoalteromonas tunicata has anti-fungal activity (Egan et al, 2002) which was later identified as a tambjamine (4-methoxypyrrolecontaining bioactive compounds) like alkaloid. These tambjamines have been isolated from marine invertebrates and have been previously reported to possess antimicrobial, antitumorigenic, immunosuppressive, and anti-proliferative and ichthyodeterrent activities (Schmitt et al, 1991).

Bacterial polysaccharides are considered as pharmaceutically important secondary metabolites which are found that exopolysaccharides (EPS) from marine bacteria contain novel formulations with a variety of properties like thickening, coagulating, adhesion, stabilizing and gelling, which makes them suitable candidates for industrial applications. An EPS-2 having immunomodulatory and anti viral effects on immunocompetent cells, has been reported from Geobacillus thermodenitrificans (Arena et al, 2009).

\section{Marine fungi:-}

Fungi derived from marine sources are considered to represent a huge reservoir of secondary metabolites, many of which are biologically active. Most of these metabolites are reported to be produced by multifunctional enzyme complexes such as polyketide synthases (PKS) and non-ribosomal peptide synthetases (NRPS) (Gallo et al, 2013). Marine fungi are highly potent producers of bioactive substances with antifungal, antibacterial, antiviral, cytotoxic and immunosuppressive activity. The various biological activities make them a valuable source for pharmaceutical applications (Newman et al, 2006). Since most of marine fungi are endophilic or ephiphilic, specific methods should be followed to isolate and cultivate from various marine organisms (sponges, algae and mangrove plants) in order to characterize and elucidate the structure of secondary metabolites produced by these fungi (Kjer et al, 2010). However, isolation of bioactive compounds from marine fungi remains continuing. A marine filamentous fungus, Aspergillus glaucus extract exhibit cytotoxicity against K562 and P388 cell lines (Du et al, 2007). Antitumor activities of alkaloids isolated from a Penicillium $s p$. derived from deep ocean sediment were also reported (Bhatnagar and Kim, 2010). Weak cytotoxicity was reported from compounds isolated from Aspergillus glaucus (Du et al, 2010).

A novel antimitotic and antifungal substances were reported from low concentration of the ethanolic extracts of the culture broth of a Fusarium sp. (Mizuno et al, 2013), while a novel antibacterial dioxopiperazine, dehydroxybisdethiobis-methylthio-gliotoxin, and the previously reported bisdethiobis-methylthio-gliotoxin and gliotoxin, were isolated from the broth of a marine derived fungus of the genus Pseudallescheria. These compounds exhibited potent antibacterial activity against the methicillin resistant and multidrug resistant Staphylococcus aureus, whereas Gliotoxin showed a significant radical scavenging activity against 1, 1-diphenyl-2-picrylhydrazyl (DPPH) with $\mathrm{IC}_{50}$ value of $5.2 \mu \mathrm{M}$ ( $\mathrm{Li}$ et al, 2006). A vast number of bioactivities are reported from sponge associated fungi such as anti biotic activities ( $\mathrm{Li}$ et al, 2015) while bioactive compounds were found from gorgonian derived Cladosporium sp (Xu et al, 2015). Anti cancer compounds such as Scopularide A was reported from Scopulariopsis brevicaulis (Lukassen et al, 2015).Anti bacterial and anti fungal activities (Hong et al, 2015) were also reported from marine fungi.

Nature continues to be most important resource in the providing of novel bioactive compounds which are important in treating various human ailments. Though a considerably a high number of bioactive compounds have been isolated from marine actinomycetes, bacteria and fungi, yet there is much hidden unexplored marine diversity of these organisms remain untapped. The rapid progress in genomic information with simultaneous advances in science and marine biotechnology would definitely stimulate the search for secondary metabolite producers and secondary metabolite biosynthesis. It will undoubtedly will greatly improve our knowledge on the potential of secondary metabolite production in marine bacterial fungi and thus will provide a great opportunity to meet the future challenges in marine drug discovery. 


\section{References:-}

1. Arena, A., Gugliandolo, C., Stassi, G., Pavone, B., Iannello, D., Bisignano, G., \& Maugeri, T. L. (2009). An exopolysaccharide produced by Geobacillus thermodenitrificans strain B3-72: antiviral activity on immunocompetent cells. Immunology letters, 123(2), 132-137.

2. Barbachyn, M. R., \& Ford, C. W. (2003). Oxazolidinone structure-activity relationships leading to linezolid. Angewandte Chemie International Edition, 42(18), 2010-2023.

3. Bhatnagar, I., \& Kim, S. K. (2010). Immense essence of excellence: marine microbial bioactive compounds. Marine drugs, 8(10), 2673-2701.

4. Charan, R.D., Garson, M.J., Brereton, I.M., Willis, A.C., Hooper, J.N.A., 1996. Haliclonacylamins A and B: cytotoxic alkaloids from the tropical marine sponges Haliclona sp. Tetrahedron 52, $9111-9120$

5. Cragg, G. M., \& Newman, D. J. (2013). Natural products: a continuing source of novel drug leads. Biochimica et Biophysica Acta (BBA)-General Subjects, 1830(6), 3670-3695.

6. Debbab, A., Aly, A. H., Lin, W. H., \& Proksch, P. (2010). Bioactive compounds from marine bacteria and fungi. Microbial biotechnology, 3(5), 544-563.

7. Egan, S., James, S., Holmström, C., \& Kjelleberg, S. (2002). Correlation between pigmentation and antifouling compounds produced by Pseudoalteromonas tunicata. Environmental Microbiology, 4(8), 433-442.

8. Jensen, P. R., Gontang, E., Mafnas, C., Mincer, T. J., \& Fenical, W. (2005). Culturable marine actinomycete diversity from tropical Pacific Ocean sediments. Environmental microbiology, 7(7), 1039-1048.

9. Jha, R. K., \& Zi-Rong, X. (2004). Biomedical compounds from marine organisms. Marine drugs, 2(3), 123146.

10. Maldonado, L. A., Stach, J. E., Pathom-aree, W., Ward, A. C., Bull, A. T., \& Goodfellow, M. (2005). Diversity of cultivable actinobacteria in geographically widespread marine sediments. Antonie Van Leeuwenhoek, 87(1), 11-18.

11. Oldfield, C., Wood, N.T., Gilbert, S.C., Murray, F.D., and Faure, F.R. 1998. Desulphurisation of benzothiophene and dibenzothiophene by actinomycete organisms belonging to the genus Rhodococcus, and related taxa. Antonie Van Leeuwenhoek. 74: 119-132.

12. Romanenko, L. A., Uchino, M., Kalinovskaya, N. I., \& Mikhailov, V. V. (2008). Isolation, phylogenetic analysis and screening of marine mollusc-associated bacteria for antimicrobial, hemolytic and surface activities. Microbiological research, 163(6), 633-644.

13. Schmitt, T. M., Lindquist, N., \& Hay, M. E. (1998). Seaweed secondary metabolites as antifoulants: effects of Dictyota spp. diterpenes on survivorship, settlement, and development of marine invertebrate larvae. Chemoecology, 8(3), 125-131

14. Strohl, W. R. (2004). Antimicrobials. In Microbial diversity and bioprospecting (pp. 336-355). American Society of Microbiology.

15. Zhang, L., An, R., Wang, J., Sun, N., Zhang, S., Hu, J., \& Kuai, J. (2005). Exploring novel bioactive compounds from marine microbes. Current opinion in microbiology, 8(3), 276-281.

16. Berdy,J (2005). Bioactive microbial metabolites. Journal of Antibiotics, 58(1): 1-26

17. Lam, K. S. (2006). Discovery of novel metabolites from marine actinomycetes. Current opinion in microbiology,Elsivier (2006, 9:245-251)

18. D Chauhan,D, Hideshima,T and Anderson, K.C. (2006). A novel proteasome inhibitor NPI0052 as an anticancer therapy. British Journal of Cancer ; 95(8): 961-965.

19. Maldonado,L.A., James E.M. Stach, Wasu Pathom-aree, Alan C. Ward, Alan T. B and Michael G (2005).Diversity of cultivable actinobacteria in geographically widespread marine sediments, Antonie van Leeuwenhoek 87:11-18.

20. Gallo, M.L., Seldes, A.M. and Cabrea, G.M. (2003). Antibiotic long-chain A,B-unsaturated Aldehydes from the culture of the marine fungus Cladosporium sp. Biochemicals systematic and Ecology.32(6),545-551)

21. Newman, D. J., \& Hill, R. T. (2006). New drugs from marine microbes: the tide is turning. Journal of Industrial Microbiology and Biotechnology, 33(7), 539-544.

22. Kjer, J., Debbab, A., Aly, A. H., \& Proksch, P. (2010). Methods for isolation of marine-derived endophytic fungi and their bioactive secondary products. Nature protocols, 5(3), 479.

23. Du, Z., Li, H., \& Gu, T. (2007). A state of the art review on microbial fuel cells: a promising technology for wastewater treatment and bioenergy. Biotechnology advances, 25(5), 464-482.

24. Mizuno, C. M., Rodriguez-Valera, F., Kimes, N. E., \& Ghai, R. (2013). Expanding the marine virosphere using metagenomics. PLoS genetics, 9(12), e1003987. 
25. Li, Z. Y., \& Liu, Y. (2006). Marine sponge Craniella austrialiensis-associated bacterial diversity revelation based on 16S rDNA library and biologically active Actinomycetes screening, phylogenetic analysis. Letters in applied microbiology, 43(4), 410-416.

26. Li, Z. Y., He, L. M., Wu, J., \& Jiang, Q. (2006). Bacterial community diversity associated with four marine sponges from the South China Sea based on 16S rDNA-DGGE fingerprinting. Journal of Experimental Marine Biology and Ecology, 329(1), 75-85.

27. Xu, L., Meng, W., Cao, C., Wang, J., Shan, W., \& Wang, Q. (2015). Antibacterial and antifungal compounds from marine fungi. Marine drugs, 13(6), 3479-3513.

28. Lukassen, M. B., Saei, W., Sondergaard, T. E., Tamminen, A., Kumar, A., Kempken, F., ... \& Sørensen, J. L. (2015). Identification of the scopularide biosynthetic gene cluster in Scopulariopsis brevicaulis. Marine drugs, 13(7), 4331-4343. 